\title{
THE COEFFICIENTS OF THE RECIPROCAL OF A BESSEL FUNCTION ${ }^{1}$
}

L. CARLITZ

Put

$$
\left\{\sum_{n=0}^{\infty} \frac{(-1)^{n} x^{n}}{n ! n !}\right\}^{-1}=\sum_{n=0}^{\infty} \frac{\omega_{n} x^{n}}{n ! n !} .
$$

This is equivalent to

$$
\sum_{r=0}^{n}(-1)^{r}\left(\begin{array}{l}
n \\
r
\end{array}\right)^{2} \omega_{r}= \begin{cases}1 & (n=0) \\
0 & (n>0) .\end{cases}
$$

In a letter to the author, J. Riordan has raised the question whether the $\omega_{n}$ can satisfy a recurrence of order independent of $n$. We shall show that the $\omega_{n}$ cannot satisfy a recurrence order $k$, where $k$ is independent of $n$, with polynomial coefficients. More precisely we show that the assumption

$$
\sum_{j=0}^{k} A_{j}(n) \omega_{n+j}=0 \quad(n>N),
$$

where the $A_{j}(n)$ are polynomials in $n$ with complex coefficients and $k, N$ are fixed, leads to a contradiction.

Since it is no more difficult, we consider the following more general problem. Put

$$
\left\{\sum_{n=0}^{\infty} \frac{(-1)^{n} x^{n}}{n ! \Gamma(\nu+n+1)}\right\}^{-1}=\sum_{n=0}^{\infty} \frac{\omega_{n}(\nu) x^{n}}{n ! \Gamma(\nu+n+1)} .
$$

This is equivalent to

$$
\sum_{r=0}^{n}(-1) r\left(\begin{array}{c}
\nu+n \\
r
\end{array}\right)\left(\begin{array}{c}
\nu+n \\
n-r
\end{array}\right) \omega_{r}(\nu)= \begin{cases}1 & (n=0) \\
0 & (n>0) .\end{cases}
$$

We assume that $\nu$ is not a negative integer; then it is clear that the $\omega_{n}(\nu)$ are uniquely determined by (3) or (4).

Now assume that the $\omega_{n}(\nu)$ satisfy the recurrence

$$
\sum_{j=0}^{k} A_{j}(n, \nu) \omega_{n+j}(\nu)=0
$$

Received by the editors January 22, 1963.

1 Supported in part by National Science Foundation grant G-16485. 
for all $n>N$, where the $A_{j}(n, \nu)$ are polynomials in $n$ with complex coefficients and $k, N$ are fixed. Put

$$
\begin{aligned}
& f(x)=\sum_{n=0}^{\infty} \frac{(-1)^{n} x^{n}}{n ! \Gamma(\nu+n+1)}, \\
& g(x)=\frac{1}{f(x)}=\sum_{n=0}^{\infty} \frac{\omega_{n}(\nu) x^{n}}{n ! \Gamma(\nu+n+1)} .
\end{aligned}
$$

Now if $P(x)$ is an arbitrary polynomial with constant coefficients, it is evident that

$$
P(x D) g(x)=\sum_{n=0}^{\infty} P(n) \frac{\omega_{n}(\nu) x^{n}}{n ! \Gamma(\nu+n+1)},
$$

where $D=d / d x$; moreover since

$$
D^{j} g(x)=\sum_{n=0}^{\infty} \frac{\omega_{n+j}(\nu) x^{n}}{n ! \Gamma(\nu+n+j+1)},
$$

it follows that

$$
P(x D) \cdot D^{i} g(x)=\sum_{n=0}^{\infty} \frac{P(n)}{(\nu+n+1)_{j}} \frac{\omega_{n+j}(\nu) x^{n}}{n ! \Gamma(\nu+n+1)} .
$$

If we multiply both sides of (5) by

$$
\frac{x^{n}}{n ! \Gamma(\nu+n+1)}
$$

and sum over all $n>N$ we get

$$
\sum_{j=0}^{k} \sum_{n=0}^{\infty} A_{j}(n, \nu) \frac{\omega_{n+j}(\nu) x^{n}}{n ! \Gamma(\nu+n+1)}=C(x),
$$

where $C(x)$ is a polynomial in $x$ of degree $\leqq N$. Repeated differentiation of (7) leads to an equation of the same kind in which the right member vanishes.

Comparison of (7) with (6) shows that $g(x)$ satisfies a differential equation of the form

$$
\sum_{j=0}^{m} B_{j}(x, \nu) D^{m-j} g(x)=0,
$$

where the $B_{j}(x, \nu)$ are polynomials in $x$. The order $m$ depends upon the degree of the $A_{j}(n, \nu)$. We may assume that

$$
B_{0}(x, \nu) \neq 0 \text {. }
$$


In the next place since $g(x)=1 / f(x)$, we have

$$
\begin{aligned}
& g^{\prime}(x)=-\frac{f^{\prime}(x)}{f^{2}(x)}, \quad g^{\prime \prime}(x)=-\frac{f^{\prime \prime}(x)}{f^{2}(x)}+\frac{2\left(f^{\prime}(x)\right)^{2}}{f^{3}(x)}, \\
& g^{\prime \prime \prime}(x)=-\frac{f^{\prime \prime \prime}(x)}{f^{2}(x)}+6 \frac{f^{\prime}(x) f^{\prime \prime}(x)}{f^{3}(x)}-6 \frac{\left(f^{\prime}(x)\right)^{3}}{f^{4}(x)},
\end{aligned}
$$

and so on. Making use of (10) we may replace (8) by a differential equation in $f(x)$.

For simplicity we shall assume $m=3$; the method is however quite general. We find that

$$
\begin{aligned}
& B_{0}\left\{-f^{2}(x) f^{\prime \prime \prime}(x)+6 f(x) f^{\prime}(x) f^{\prime \prime}(x)-6\left(f^{\prime}(x)\right)^{3}\right\} \\
& +B_{1}\left\{-f^{2}(x) f^{\prime \prime}(x)+2 f(x)\left(f^{\prime}(x)\right)^{2}\right\}-B_{2} f^{2}(x) f^{\prime}(x)+B_{3} f^{3}(x)=0
\end{aligned}
$$

where $B_{j}=B_{j}(x, \nu)$. Now, on the other hand, we have

$$
x f^{\prime \prime}(x)+(\nu+1) f^{\prime}(x)+f(x)=0,
$$

so that

$$
x f^{\prime \prime \prime}(x)+(\nu+2) f^{\prime \prime}(x)+f^{\prime}(x)=0 .
$$

We may eliminate $f^{\prime \prime}(x)$ and $f^{\prime \prime \prime}(x)$ in (11); there results an equation of the form

$$
\begin{aligned}
C_{0}(x, \nu)\left(f^{\prime}(x)\right)^{3}+C_{1}(x, \nu) & \left(f^{\prime}(x)\right)^{2} f(x) \\
& +C_{2}(x, \nu) f^{\prime}(x) f^{2}(x)+C_{3}(x, \nu) f^{3}(x)=0,
\end{aligned}
$$

where $C_{j}(x, \nu)$ are polynomials in $x$. Moreover, by (9) $C_{0}(x, \nu)$ $=-6 B_{0}(x, \nu) \neq 0$.

It therefore follows from (12) that $f^{\prime}(x) / f(x)$ is an algebraic function of $x$. However, since $f(x)$ has infinitely many zeros, it follows that the logarithmic derivative $f^{\prime}(x) / f(x)$ has infinitely many poles and therefore cannot be an algebraic function.

We have proved the following

THEOREM. Let $\nu$ be an arbitrary complex number not equal to a negative integer and define $\omega_{n}(\nu)$ by means of (3). Then $\omega_{n}(\nu)$ cannot satisfy a recurrence

$$
\sum_{j=0}^{k} A_{j}(n, \nu) \omega_{n+j}(x)=0 \quad(n>N),
$$

where the $A_{j}(n, \nu)$ are polynomials in $n$ with complex coefficients and $k$, $N$ are fixed.

DUKE UNIVERSITY 